endothelial growth factor, hepatocyte growth factor or stem cell-derived factor-1, were not significantly different among the groups, indicating that angiogenic process might have been completed at the 4 weeks after the treatment.

Conclusions ONO-1301SR combined with omental flap therapy promoted myocardial angiogenesis with collateral artery growth, leading to recovery of the cardiac function in a porcine ICM model. This combined therapy may be useful in regenerating myocardial microvasculature.

\section{FUNCTIONAL ROLE OF MICRORNA-214 IN MODULATING VASCULAR SMOOTH MUSCLE CELL FUNCTIONS AND NEOINTIMA FORMATION}

Tayyab Adeel Afzal*, Qingzhong Xiao. Queen Mary University of London; *Presenting Author

10.1136/heartjnl-2016-309890.173

Background/Objective Vascular smooth muscle cell (VSMC) proliferation and migration play a pronounced role in the pathophysiology of atherosclerosis and neointima formation. microRNAs are short, single stranded, non-coding 22 nucleotide RNAs involved in post transcriptional gene silencing. Increasing evidence has suggested an important role of miRNAs in regulating VSMC functions. However, little is known about the functional involvements of miR-214 in VSMC proliferation/migration and vessel injury-induced neointima formation. In the current study, we aimed to study the functional importance of miR-214 and its target genes in VSMC proliferation, migration and neointima SMC hyperplasia.

Methods and Results miR-214 expression was closely associated with VSMC phenotypic modulation in response to different pathological stimuli. miR-214 over-expression in serumstarved VSMCs significantly decreased VSMC proliferation and migration, while knockdown of miR-214 dramatically increased VSMC proliferation and migration, respectively. Proteomic analyses pinpointed that NCK-associated protein 1 (NCKAP1), a major component of the WAVE complex that regulates lamellipodia formation and cell motility, was negatively regulated by miR-214 in VSMCs. NCKAP1 has also been predicted as one of the top targets of miR-214 by using several computational miRNA target prediction tools, and was indeed negatively regulated by miR-214 in VSMCs. Luciferase assay showed miR-214 substantially repressed wild type, but not the miR-214 binding site mutated version of NCKAP1$3 \hat{a} €{ }^{\text {TM }}$-UTR-luciferase activity in VSMCs, confirming the NCKAP1 is the functional target of miR-214 in VSMCs. Data from co-transfection experiments also revealed that inhibition of NCKAP1 is required for miR-214-mediated lamellipodia formation and cell motility. Importantly, locally enforced expression of miR-214 in the injured vessels significantly reduced NCKAP1 expression levels, inhibited VSMC proliferation, and prevented neointima SMC hyperplasia after injury.

Conclusions/implications We have uncovered an important role of miR-214 in modulating VSMC functions and neointima hyperplasia. Our findings have suggested that miR-214 represents a potential therapeutic target for vascular diseases.

\section{HNRNPA1 IS A CRITICAL REGULATOR IN VASCULAR SMOOTH MUSCLE CELL FUNCTIONS AND NEOINTIMA HYPERPLASIA}

Weiwei AN*, Qingzhong Xiao. Queen Mary University of London; *Presenting Author

\subsection{6/heartjnl-2016-309890.174}

Background RNA binding protein heterogeneous nuclear ribonucleoprotein A1 (hnRNPA1) plays various roles in transcriptional and posttranscriptional modulation of gene expression. Our previous study has demonstrated for the first time that hnRNPA1 plays an important role in vascular smooth muscle cell (VSMC) differentiation from stem cells in vitro and in vivo. However, little is known about the functional involvements of hnRNPA1 in VSMC functions and neointima hyperplasia.

Purpose In this study, we aimed to investigate the functional roles of hnRNPA1 in the contexts of VSMC functions, injuryinduced vessel remodelling and human atherosclerotic lesions, and the molecular mechanisms involved.

Methods and results Studies used mouse aorta VSMCs showed that hnRNPA1 expression levels were consistently modulated during VSMC phenotype switching. Moreover, VSMCs with hnRNPA1 knockdown had an increased migratory and proliferative ability, but a reduced VSMC-specific gene expression level. Consistently, over-expression of hnRNPA1 significantly reduced VSMC migration and proliferation. Furthermore, our data shows that hnRNPA1 exerts its effects on VSMC functions through modulating IQ motif containing GTPase activating protein 1 (IQGAP1) gene, a well-known important regulator of VSMC migration and proliferation. Mechanistically, hnRNPA1 regulates IQGAP1 mRNA degradation through two mechanisms: upregulating miR-124 and AU-rich element (ARE). Further evidence suggests that hnRNPA1 up-regulates miR-124 through regulating miR-124 biogenesis, and that IQGAP1 is the authentic target gene of miR-124. Importantly, the expression levels of hnRNPA1 were significantly downregulated during neointimal lesion formation induced by wire injury, suggesting a role for hnRNPA1 in vessel injury-induced neointimal development and progression. In accordance, perivascular ectopic overexpression of hnRNPA1 greatly reduced VSMC proliferation, and inhibited neointima formation in wire-injured carotid arteries. Translationally and consistently, lower expression levels of hnRNPA1 and miR-124, while higher expression levels of IQGAP1, were observed in human atherosclerotic lesions.

Conclusions We have identified hnRNPA1 as a critical regulator in VSMC functions and neointima hyperplasia. Our data provide new insight into the roles of hnRNPA1/miR-124/ IQGAP1 regulatory axis in VSMC functions and the pathogenesis of neointima formation and/or angiographic restenosis, and aid the development of novel therapeutic agents for the prevention of these diseases. 